\title{
Molecular profiling and computational network analysis of TAZ-mediated mammary tumorigenesis identifies actionable therapeutic targets
}

\author{
Costa Frangou ${ }^{1, *}$, Ying-Wei $\mathrm{Li}^{1, *}$, He Shen ${ }^{1}$, Nuo Yang ${ }^{1}$, Kayla E. Wilson ${ }^{1}$, \\ Maxime Blijlevens ${ }^{1}$, Jin Guo ${ }^{1}$, Norma J. Nowak ${ }^{2}$, Jianmin Zhang ${ }^{1}$ \\ ${ }^{1}$ Department of Cancer Genetics, Roswell Park Cancer Institute, Buffalo, NY, 14263, USA \\ ${ }^{2}$ Department of Biochemistry, School of Medicine and Biomedical Sciences, University at Buffalo, The State University of \\ New York, Buffalo, NY, 14260, USA \\ *These authors contributed equally to this work \\ Correspondence to: \\ Jianmin Zhang, e-mail: jianmin.zhang@roswellpark.org \\ Keywords: breast cancer, TAZ, tumor-initiating cells, RNA sequencing, Dasatinib \\ Received: August 07, 2014 \\ Accepted: October 03, 2014 \\ Published: October 24, 2014
}

\section{ABSTRACT}

Triple-negative breast cancer (TNBC) accounts for approximately 15-20\% of all breast cancer (BC) cases and contributes disproportionately to BC mortality. TAZ, a key transducer of the Hippo pathway, has recently been demonstrated to confer breast cancer stem cell (CSC) traits. However, TAZ target genes and the underlying transcriptional regulatory pathways responsible for the CSC phenomenon remain unknown. Here, we demonstrate that the oncogenic activity of TAZ is essential for propagation of the malignant phenotype. We further show that constitutively active TAZ tumor-derived cells exhibit unique tumor-initiating properties, including increased self-renewal and metastatic seeding potential, acquired chemotherapy resistance and the ability to efficiently regenerate tumor formation in vivo. Combined digital RNA expression analysis and computational network approaches identify several signaling pathways that distinguish breast cancer tumor-initiating cells (T-ICs) from bulk tumor cells. We demonstrate the utility of this approach by repositioning the small molecule tyrosine kinase inhibitor, Dasatinib, which selectively targets T-ICs and inhibits TNBC growth in vivo.

\section{INTRODUCTION}

The Hippo pathway is an evolutionary conserved regulator of tissue growth and cell fate during mammalian development and regeneration, while deregulation of the Hippo pathway caused by gene mutation or anomalous protein expression has been linked to human diseases including cancer $[1,2]$. Currently, over 20 regulators have been identified that intersect with core Hippo pathway components and potentially contribute to carcinogenesis. For example, loss of Neurofibromin2 (NF2) tumor suppressor function has been associated with the development of colorectal, hepatocellular and thyroid carcinomas, as well as in melanoma [3]. Central to the Hippo pathway are two key downstream effector proteins, YAP (Yes-associated protein) and TAZ (transcriptional co-activator with PDZ-binding motif) that are tightly regulated by a number of upstream signaling molecules, such as Mst1/2, Lats1/2 and RASSF family proteins $[4,5]$. Inactivation of YAP/TAZ by the Hippo pathway is mediated primarily via cytoplasmic sequestration from 14-3-3 binding and protein degradation, respectively [6-9]. In addition, Hippo pathway-independent restriction of YAP/TAZ mediated by angiomotin, scribble, PTPN14, $\alpha$-catenin and other junction protein sequestration mechanisms has been reported [10-15].

TAZ does not contain an intrinsic DNA-binding domain, but instead actively recruited to target genes by interacting with multiple transcription factors and mediates diverse transcriptional programs in a contextdependent manner [16]. Interestingly, loss of the TAZ protein resulted in uncontrolled differentiation of 
human embryonic stem cells (hESCs) as well as loss of self-renewal of hESCs [17]. Furthermore, TAZ was recently shown to sustain self-renewal potential and tumorinitiation capacities of breast CSCs [11]; however, TAZ target genes and the underlying transcriptional regulatory pathways responsible for the CSC phenomenon remain poorly characterized. Nonetheless, therapeutic modulation of TAZ could improve current cancer treatment strategies.

Unfortunately, direct pharmacological inhibition of TAZ is challenging because it has no known catalytic activity [18, 19]. Accordingly, in the current study we employ a strategy that exploits the functional interconnectivity of intracellular signaling networks to unambiguously identify disease-specific 'druggable' targets, located downstream of TAZ. Moreover, we clearly demonstrate that expression of constitutively active TAZ experimentally confers BC T-IC properties and metastatic colonization capacity to non-transformed human basal-like mammary epithelial cells. Notably, we present a novel differential network-based framework to detect biologically meaningful cancer-related genes and subsequently prioritize/rank genes as potential drug targets. Finally, we demonstrate that Dasatinib, a Src family kinase and receptor tyrosine kinase (RTK) inhibitor, selectively targets TAZ-induced T-ICs and provides novel insight to how modulation of TAZ-driven pro-tumorigenic transcriptional programs could help guide future $\mathrm{BC}$ treatment strategies.

\section{RESULTS}

\section{In vivo-derived TAZ-induced tumor initiating cells (T-ICs)}

TAZ has been previously indicated to serve as a key breast CSC determinant [11]. Accordingly, to further characterize TAZ-induced CSC-like traits during mammary tumorigenesis, we orthotopically injected TAZ-4SA (constitutively activated form) transduced MCF10A cells into the mammary fat pad of SCID mice and generated primary tumors. We explanted the TAZ-induced mammary tumors and derived cell lines (herein denoted TAZ-M\#1-6) that could be stably propagated in tissue culture (Fig. 1A). We confirmed ectopic TAZ expression in all tumor-derived cells as well as in the parental cell line by immunoblot (Fig. 1B). We previously showed that overexpression of constitutively active TAZ promoted cell migration [20]. To independently test whether tumor-derived cell lines maintained such capacity, we performed transwell cell migration assays and found that they all migrated aggressively (Fig. 1C).

A subpopulation $\mathrm{CD} 44^{\text {high }} / \mathrm{CD} 24^{\text {low }}$ of breast cancer cells has been reported to have stem/progenitor cell properties [21]. To estimate the proportion of CD44 high/ CD24 ${ }^{\text {low }}$ cells, we characterized tumor-derived cell lines by flow cytometry for surface expression of CD44 and CD24, respectively. As shown in Figure 1D, this population was indeed enriched in the majority of tumor-derived cell lines tested but phenotypically reverted to the CD44 high/ CD24 high population after extended culture (Supplemental Fig. 1A). Interestingly, the $\mathrm{CD} 44^{\text {high }} / \mathrm{CD} 24^{\text {low }}$ sub population displayed a lower in vitro proliferation rate compared to parental cells and resistance to chemotherapy (Fig. 1E, F). However, they exhibited selective sensitivity to Salinomycin [22] and Bortezomib [23] (Supplemental Fig. 1B, C), consistent with the recently reported proteasome addiction of basal-like TNBC cells [23].

Next, to determine whether the tumor-derived cells contained self-renewal capacity, we used an in vitro mammosphere formation assay and found that majority of the cell lines generated mammospheres of increased size and number (Fig. 2A). The acquisition of CSC traits has been previously associated with the epithelial to mesenchymal transition (EMT) [24]. Therefore, we further analyzed the alterations of EMT markers in the tumor derived cells. The alterations of EMT-associated markers, such as: loss of epithelial genes $\mathrm{CDH} 1, \mathrm{CDH} 3$ and gain of mesenchymal genes $\mathrm{CDH} 2$ and FN1, as well as the adoption of a migratory mesenchymal phenotype were maintained in all the tumor-derived cell lines (Fig. 1C and 2B). Interestingly, the TAZ-M\#5 cell line underwent EMT but lost the stem-like features (Fig. 1C\&D, $2 \mathrm{~A} \& \mathrm{~B}$ ) and tumor initiation potential (data not shown).

To explore whether tumor-derived CD44 high/ CD24 $4^{\text {low }}$ cells acquired tumor-initiating capacity and to accurately enumerate their frequency, we used in vivo clonal tumor initiating assays to functionally identify CSCs rather than on the basis of immunophenotype or in vitro mammosphere assay. Consequently, we estimated a $>1000$-fold increase in the frequency of self-renewing T-ICs in the TAZ-M\#1 cell line relative to the parental TAZ-4SA-expressing MCF10A cell line (Fig. 2C and data not shown). The likelihood of nodal metastases is increased in $\mathrm{BC}$ patients whose tumors have breast CSCs [21]. Furthermore, emerging evidence indicates that breast CSCs and EMT co-operate to generate circulating tumor cells (CTCs) that are highly competent for metastasis [25]. To further explore this possibility and specifically determine whether the tumor-derived cells promote metastasis, both mammary fat pad and tail-vein injections were performed in NSG mice. In contrast to control mice, both metastasis to the lungs and spontaneous metastasis were observed in NSG mice injected with tumor-derived TAZ-induced cells (TAZ-M\#1), strongly suggesting that TAZ increased metastatic potential and tumor-seeding ability (Fig. 2D).

\section{Network-based comparison of TAZ-mediated gene expression patterns}

To examine the TAZ-mediated pro-tumorigenic transcriptional program, we performed RNA-seq 
A

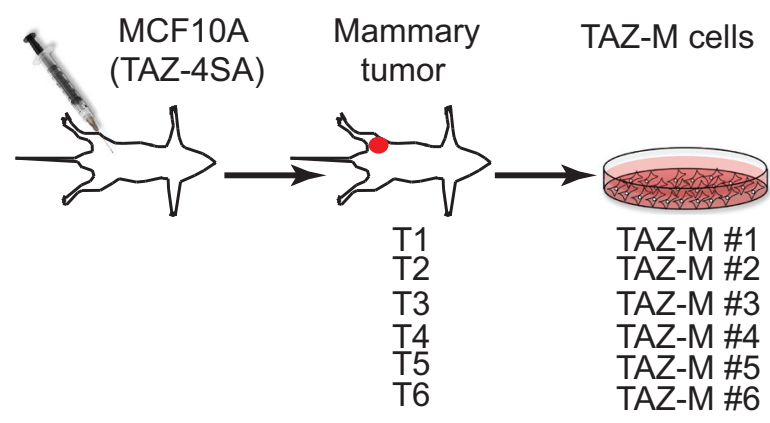

C
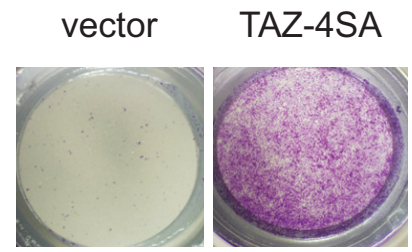

TAZ-M \#3

TAZ-M \#4
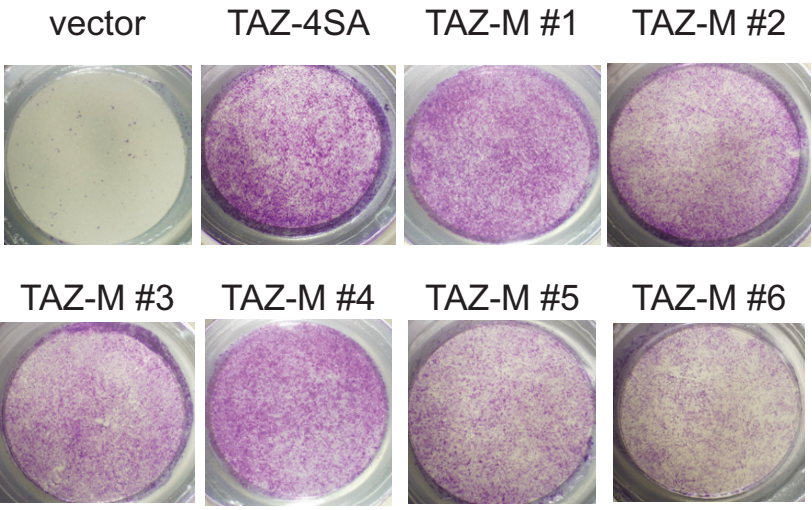

E
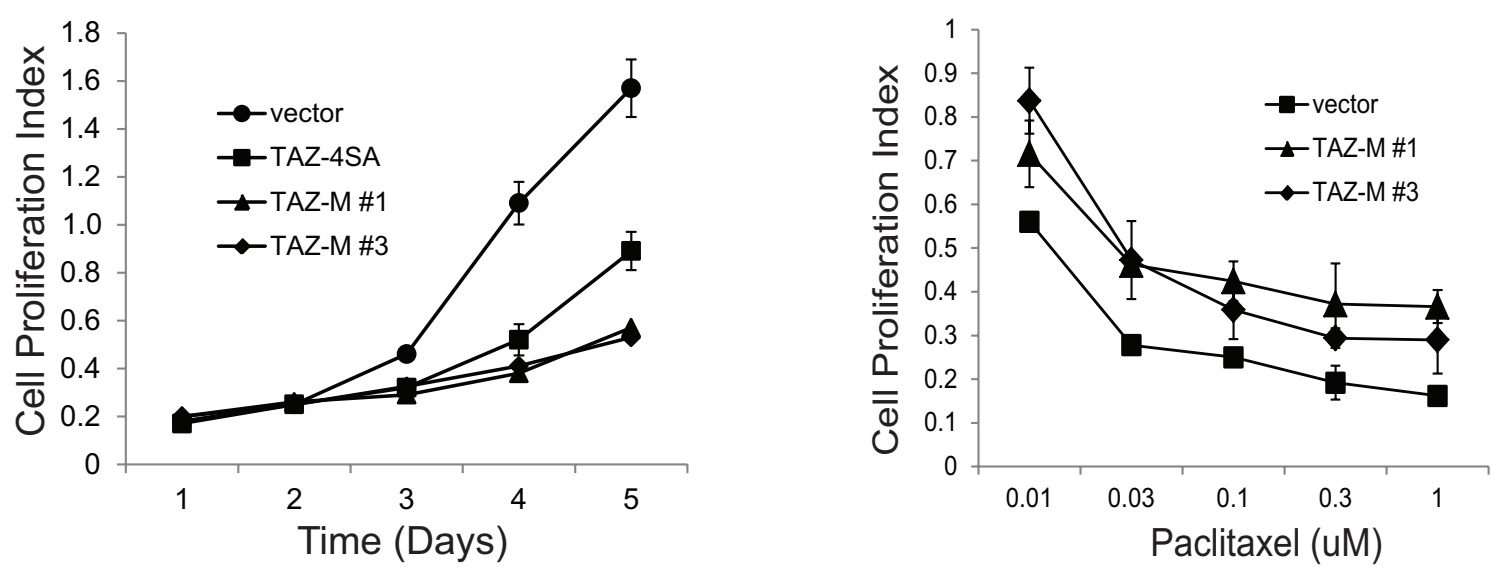

Figure 1: TAZ-induced mammary tumor derived cells exhibit a robust CSC potential. (A) Schematic description of the establishment of TAZ-4SA-derived mammary tumors and explanted cell lines. (B) Immunoblot of TAZ in the tumor-derived cells as well as in the parental TAZ-4SA MCF10A cells. $\beta$-Actin was used as the loading control. (C) Cell migration is increased in the tumor-derived cells. (D) Flow cytometry of the CD44 high/CD24 $4^{\text {low }}$ population in the tumor-derived cells as well as in the vector control or TAZ-4SA transduced MCF10A cells. (E) Proliferation curve of vector or TAZ-4SA transduced MCF10A cells, as well as TAZ-M\#1 and TAZ-M\#3 cells grown in culture. Viable cells were counted by the MTT assay. Bars denote standard errors $(n=5)$. (F) Dose-response curve of vector-transduced MCF10A cells, TAZ-M\#1 and TAZ-M\#3 cells treated with paclitaxel. Bars denote standard errors $(n=5)$.

differential gene and transcript analysis for YAP or TAZ transduced MCF10A cells, as well as a representative subset of tumor-derived cell lines (TAZ-M\#1 and
TAZ-M\#5). Comparison of the expression profiles identified 1478 genes that displayed a significant difference between samples (Fig. 3A and Supplemental Table 1). 
A

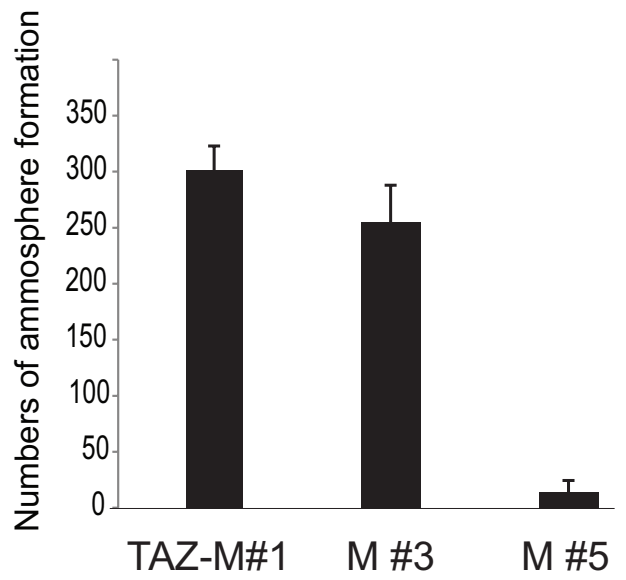

B

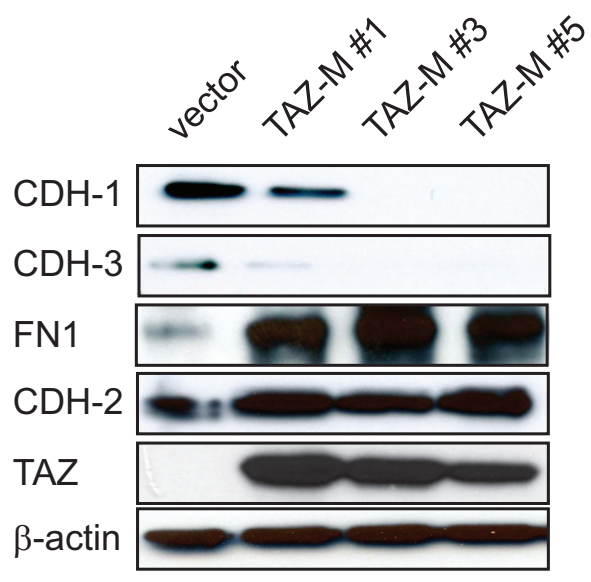

C

\begin{tabular}{|l|c|c|c|c|}
\hline Cell injected & $1 \times 10^{6}$ & $1 \times 10^{5}$ & $1 \times 10^{4}$ & $1 \times 10^{3}$ \\
\hline TAZ-M \#1 & $6 / 6$ & $6 / 6$ & $6 / 6$ & $6 / 6$ \\
\hline
\end{tabular}

D
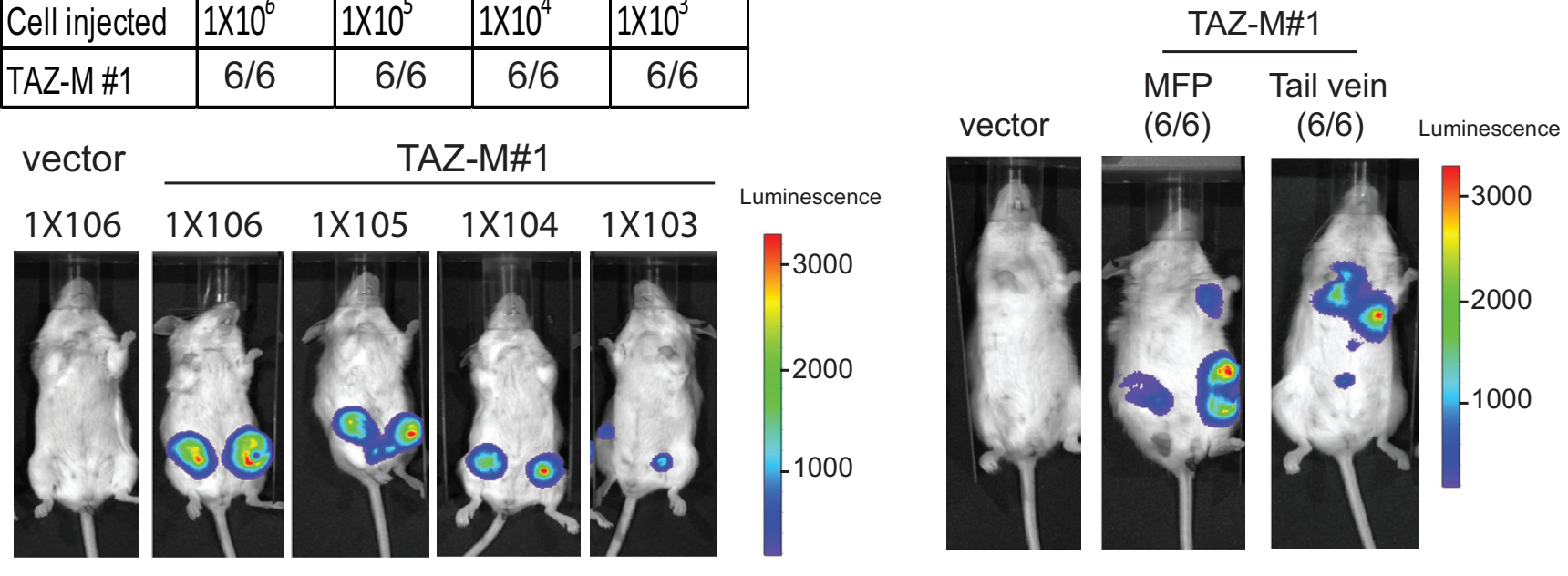

Figure 2: Tumor-derived cells acquired efficient tumor initiation and metastatic capacity. (A) Quantification of mammosphere formation in TAZ-M\#1, TAZ-M\#3 and TAZ-M\#5 cells. Bars denote standard errors ( $\mathrm{n}=5$ ). (B) TAZ-M\#1, TAZ-M\#3 and TAZ-M\#5 cells undergo EMT. Immunoblot shows decreased E-cadherin and P-cadherin (epithelial markers) and concomitantly increased $\mathrm{N}$-cadherin and fibronectin (mesenchymal markers). $\beta$-Actin was used as the loading control. (C) Tumor-seeding capacity of TAZ-M\#1 cells when injected into the mammary fat pad of NOD/SCID mice. (D) Metastatic tumor-seeding capacity of TAZ-M\#1 cells when injected into the mammary fat pad and tail vein of the NSG mice.

As shown in Figure 3B, five data subtypes were identified by consensus NMF clustering using an abundance matrix containing differentially changed genes $(>2.0$ fold change, with $p<0.05$ in all expression profiles). Using DAVID (database for annotation, visualization and discovery), we assessed whether any GO biological or molecular processes were statistically over- or underrepresented. Over-represented pathways (FDR $<0.001$ ) included EMT related pathways, along with integrin signaling and focal adhesion, chromatin remodeling, PIK3 signaling, growth hormone and nuclear receptor co-activators, WNT/cadherin signaling and ATM/Rb related pathways (Supplemental Table 2-4). In addition, Gene Set Enrichment Analysis (GSEA) of differentially regulated transcripts in the TAZ-M\#1 gene cluster/signature revealed an overrepresentation of genes associated with several published invasive, metastatic BC patient and breast CSC transcriptome studies (Supplemental Table 5).

To expand on these analyses, we constructed a functional interaction (FI) network and used network community analysis to identify sub-networks that contain modules involved in annotated biological processes and canonical pathways [26]. Specifically, two interaction networks were independently generated from the TAZ-M\#1 and TAZ-M\#5 tumor data sets. Network subtraction was subsequently used to obtain a differentialnetwork model and to identify both overlapping and unique clusters (Fig. 3C and Supplemental Table 6). Network comparison reflected differential functional interactions unique to TAZ-M\#1, a breast tumor that 
A

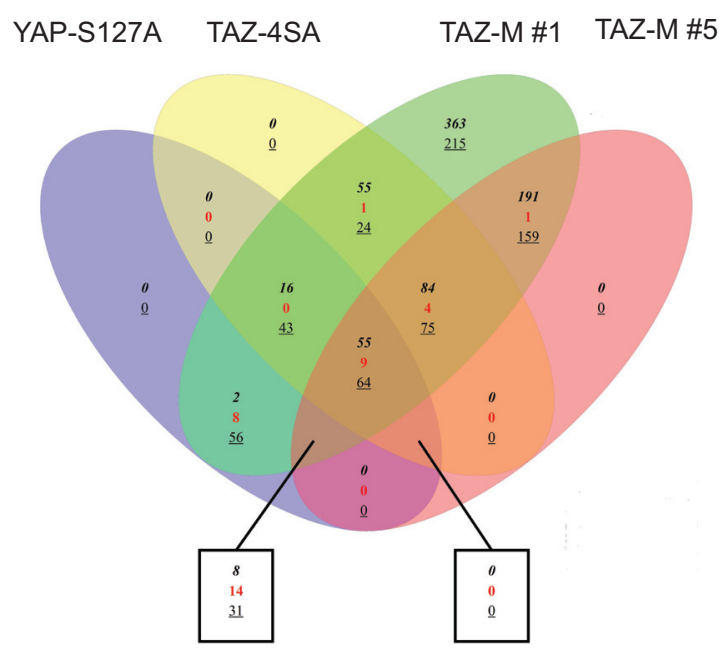

C

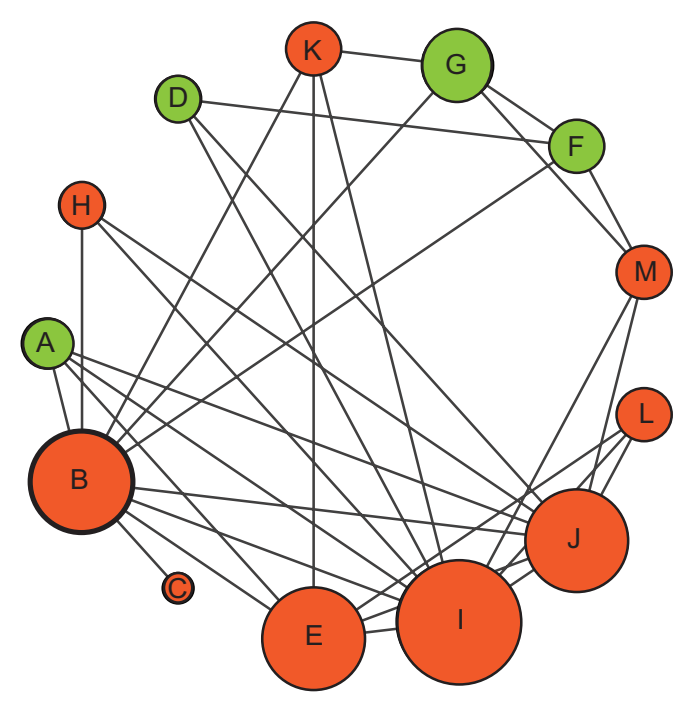

B

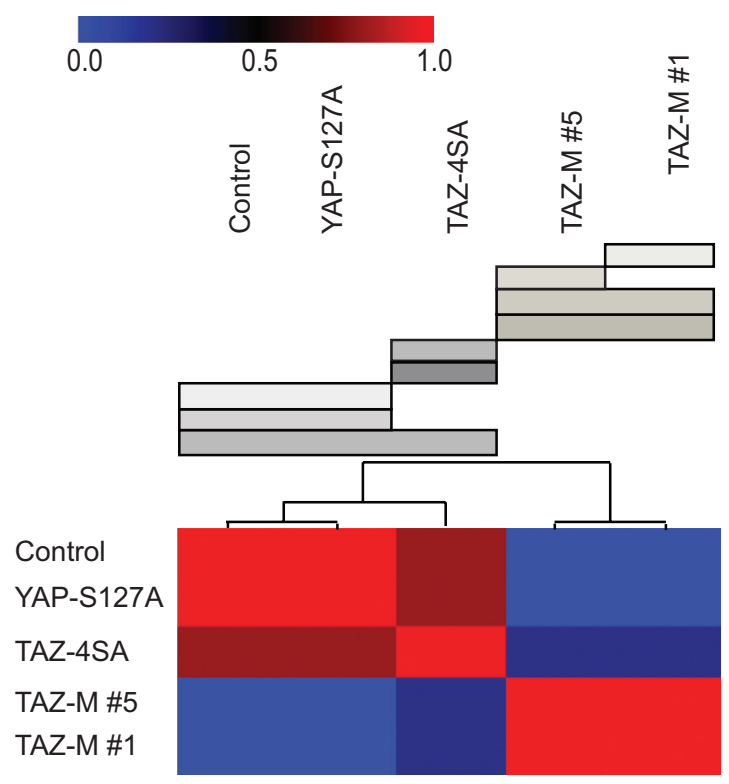

D

\begin{tabular}{|c|c|c|c|}
\hline Gene Symbol & Node Degree & Betweenness & Closeness \\
\hline PDGFRB & 56 & 32927 & $9.50 \mathrm{E}-04$ \\
\hline FYN & 71 & 31331 & $9.62 \mathrm{E}-04$ \\
\hline ACTB & 51 & 20095 & $8.73 \mathrm{E}-04$ \\
\hline HSP90AA1 & 27 & 15461 & $8.42 \mathrm{E}-04$ \\
\hline COPA & 17 & 9426 & $7.50 \mathrm{E}-04$ \\
\hline AKT1 & 24 & 8914 & $8.56 \mathrm{E}-04$ \\
\hline DUSP1 & 24 & 8855 & $7.85 \mathrm{E}-04$ \\
\hline ARF1 & 21 & 7957 & $6.63 \mathrm{E}-04$ \\
\hline CALM2 & 19 & 7755 & $7.80 \mathrm{E}-04$ \\
\hline COPB2 & 15 & 7750 & $7.08 \mathrm{E}-04$ \\
\hline LAMB3 & 36 & 7568 & $8.43 \mathrm{E}-04$ \\
\hline PIK3R1 & 37 & 7529 & $8.61 \mathrm{E}-04$ \\
\hline TMED2 & 11 & 7220 & $6.21 \mathrm{E}-04$ \\
\hline SSRP1 & 21 & 7040 & $7.67 \mathrm{E}-04$ \\
\hline CEBPB & 12 & 6562 & $7.75 \mathrm{E}-04$ \\
\hline SSB & 14 & 6508 & $6.85 \mathrm{E}-04$ \\
\hline NFKBIA & 18 & 6490 & $8.37 \mathrm{E}-04$ \\
\hline COL1A1 & 43 & 6400 & $8.18 \mathrm{E}-04$ \\
\hline
\end{tabular}

Figure 3: Identification of common and distinct TAZ target gene signatures. (A) Venn diagram describing the number of genes that changed in each condition and their relation to each other. To indicate the polarity of numerical regulation of each factor within each set or intersection, we have employed a three-way key system. The numbers of factors unique to a set, with a positive regulation polarity, are identified with an italic numeral. The numbers of factors unique to a set, with a negative regulation polarity, are identified with an underlined numeral. The numbers of factors common between multiple sets are indicated with a red colored numeral. (B) TAZ-M\#1 and TAZ-M\#5 tumors clustered using NMF expression signatures from RNA-Seq data sets and the consensus clustering matrix for the clusters are shown. Based on the visual inspection of a hierarchical clustering of the consensus matrix defining the average connectivity over 1000 clustering runs with different initial conditions, the case of $\mathrm{K}=5$ was used to arrange samples in, giving rise to 5 clusters highlighted by red and blue, respectively. (C) Graphical depiction of enriched pathway modules obtained by edge-betweenness network clustering results for the altered genes from the TAZ-M\#1 and TAZ-M\#5 functional interaction (FI) networks. Each node is a pathway and nodes are linked by directed edges representing parent-to-child relationships. Nodes are colored by red (common networks) or green (TAZ-M\#1 specific); the size of each node is proportional to the number of proteins annotated with that term. (D) Table summarizing the degree and centrality measures for the top 18 TAZ-M\#1 bottlenecks proteins that are defined as central nodes. The bottlenecks control the flow of biological information within the TAZ-M\#1 network, and their disruption can break the entire network into small components. From 18 major bottlenecks observed within the network, 5 bottlenecks represent new protein targets that are suitable for the development of TNBC. 
had acquired stem-like traits. PDGFR- $\beta$, WNT and NF-kappa B signaling pathways were highly deregulated in TAZ-M\#1 (FDR < 0.0001), and may represent key mediators and/or regulators of biological properties associated with CSCs [27].

Next, to reduce the list of candidate genes to those that are most likely associated with signaling pathways involved in self-renewal and activated in CSCs, as opposed to general cell proliferation and pro-survival mechanisms, we identified all network bottleneck proteins. These proteins are known to control the flow of biological information within a network and maintain network integrity. Briefly, we define network bottlenecks as nodes (proteins) with the highest betweenness centrality scores and estimated by calculating the number of shortest paths that cross a given node. In addition, highly connected nodes (hubs) are also known to play an integral role in the propagation of signals across a network and were also calculated (Fig. 3D \& Supplemental Methods). As summarized in Fig. 3D, top-ranked bottleneck genes are highly deregulated in BC patients and several genes, such as PDGFR- $\beta$, FYN and NF-kappaB components.

\section{Dasatinib targets the TAZ-induced T-ICs and TNBC Cells}

Data presented thus far identify TAZ as a potential new drug target to treat aggressive types of BC. Unfortunately, no pharmacological agents directly targeting active TAZ have been reported. Therefore, we rationalized that modulating 'druggable' targets downstream of TAZ, rather than the oncogene itself, could be therapeutically exploited. Therefore, we used a subtractive integrated network approach and generated a TAZ-specific bipartite graph in which every link connected all FDA-approved drugs to a protein, if the protein is a known target of the drug (i.e. drug-target network, DTN) (Supplemental Table 7). As summarized in Figure 4A, one FI sub-cluster of particular interest was highly enriched with the PDGFR- $\beta$ signaling pathway components. Furthermore, PDGFR- $\beta$, FYN and Src genes included in this module were independently identified as key bottleneck network genes (Fig. 3D).

Dasatinib is a potent, multi-target kinase inhibitor predicted from our DTN analyses (Supplemental Table 7). It was initially developed as an inhibitor of the Src family kinases (SFKs, such as Fyn, Yes, Src and Lyk) but is now known to also inhibit BCR-ABL, EphA2, platelet derived growth factor receptor (PDGFR) and c-KIT [28]. Accordingly, we rationalized that Dasatinib would represent an apposite and novel multi-target repositioning agent to pharmacologically modulate TAZ-mediated pro-tumorigenic transcriptional programs. To our great interest, we found that Dasatinib inhibited the anchorageindependent growth of TAZ-M\#1 cells in soft-agar assay and reduced self-renewal as measured by mammosphere formation (Fig. 4B, C). Remarkably, FACS analysis of Dasatinib-treated TAZ-M\#1 cells had an almost completely depleted CD44 $4^{\text {high }} / \mathrm{CD} 24^{\text {low }}$ subpopulation after drug treatment for 24 hours and recovery of 72 hours; and only the $\mathrm{CD} 44^{\text {high }} / \mathrm{CD} 24^{\text {high }} \mathrm{BC}$ subpopulation remained viable (Fig. 4D). Collectively, these results indicate that Dasatinib selectively killed the TAZ driven BC T-ICs.

TNBC frequently has enriched breast CSCs that are thought to drive tumorigenesis and contribute to maligancy, therapeutic resistance and clinical relapse [29]. To further explore whether Dasatinib specifically targets TNBC cells, we used a panel of human TNBC and luminal cell lines to further assess differential sensitivity and response to Dasatinib. First, we examined the halfmaximal inhibitory concentration (IC50) values for Dasatinib and found that TNBC cell lines were more sensitive to Dasatinib than luminal cell lines (Fig. 4E). In addition, we confirmed the effect of Dasatinib on TNBC cell line proliferation in vitro by colony formation assay (Fig. 4F). Finally, to evaluate the effect of Dasatinib on mammary tumor-forming potential in vivo, we injected either vehicle control or 24-hour low-dose (300 nM) Dasatinib treated MDA-MB-231 cells into the mammary fat pad of SCID mice and clearly demonstrated that Dasatinib decreased tumor formation capacity (Fig. 4G).

\section{DISCUSSION}

The CSC hypothesis is based on the observation that many cancers are driven by a subpopulation of tumor-initiating cells (popularly known as CSCs) and promote tumor growth. In addition, CSCs are resistant to chemotherapy and radiation treatments, which potentially explain the limitations of these agents in curing human malignancies [30-32]. Despite considerable research efforts in recent years, it remains unclear what fraction of cancers follow the CSC model as well as the clinical behaviors explained by the model. Nonetheless, delineation of essential pathways that can distinguish T-ICs from their normal counterparts could provide novel opportunities for therapeutic intervention.

Multi-omics approaches allow the characterization of biological systems in various disease states to be measured from an increasing number of biochemical and molecular perspectives. Unfortunately, conventional functional enrichment analyses are limited in their efficacy because they do not incorporate known interdependencies among genes within a pathway. Furthermore, they treat all gene alterations as equal, which is not expected to be valid for many biological systems. Conversely, network-based pathway analysis identifies markers not as individual entities but as sub-networks and the resulting sub-networks provides an effective way to visualize genes and their interactions/relationships [33]. Here, employing 
A

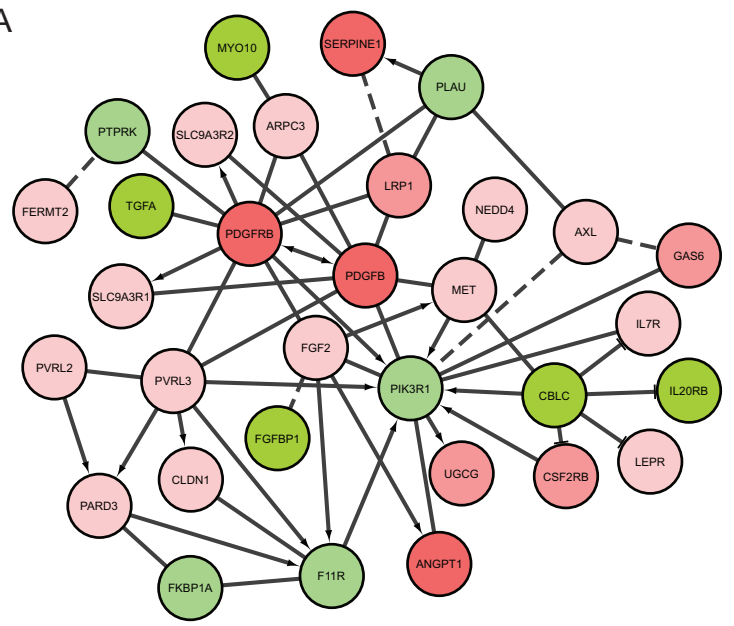

D

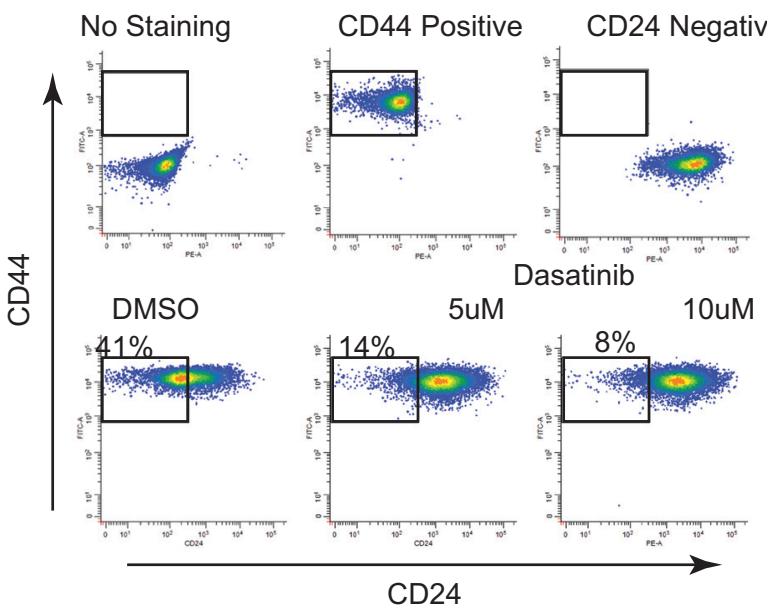

$\mathrm{F}$

\section{MDA-MB231 Cal-51 Sum159 Cal-120}
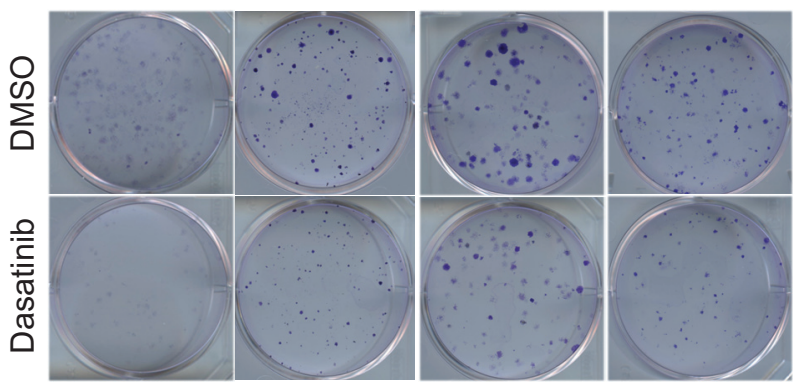

B
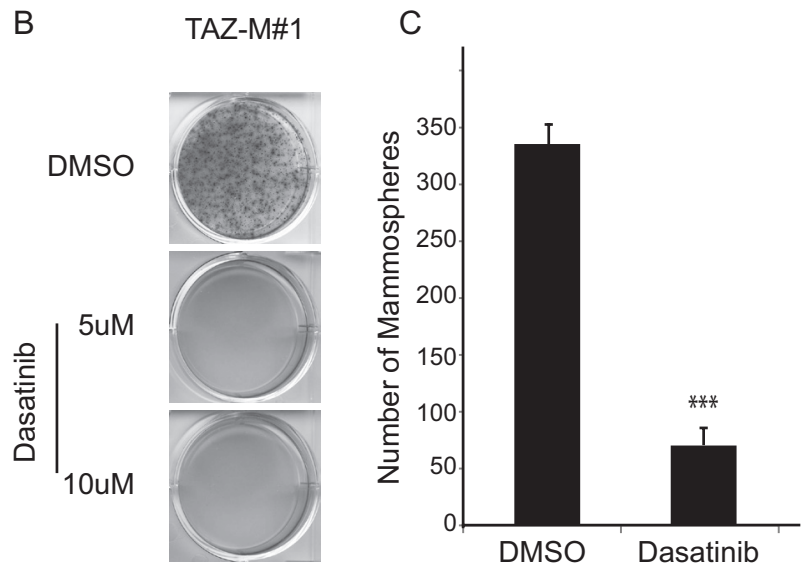

$\mathrm{E}$

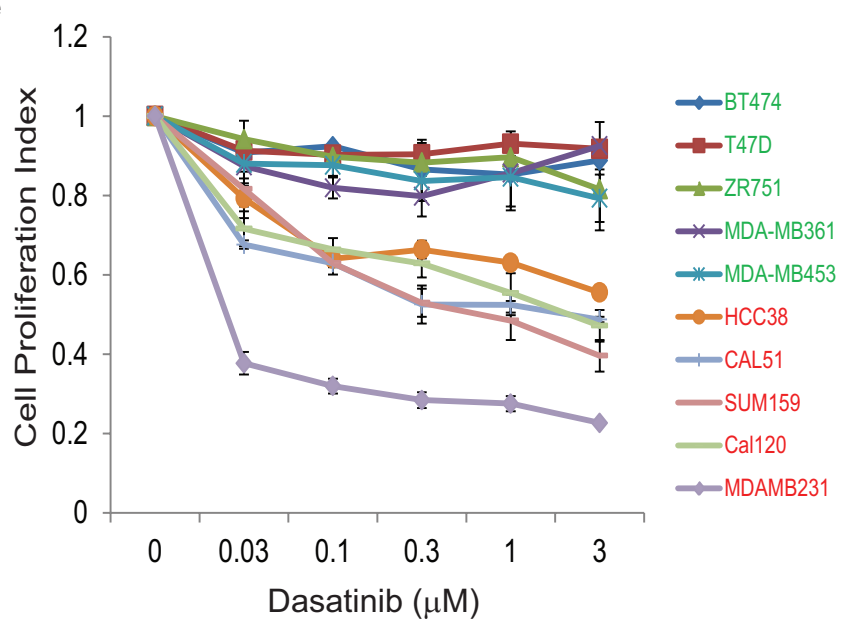

G

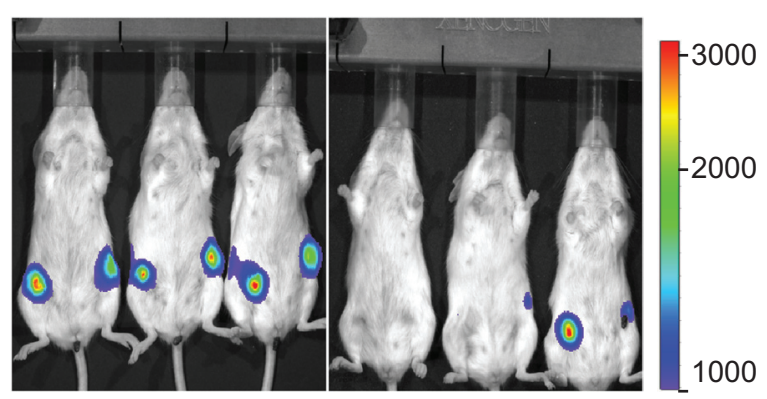

Figure 4: Identification of Dasatinib as a potent inhibitor for TAZ-induced stem-like properties and TNBC tumorigenicity. (A) PDGFR- $\beta$-centered subcluster identified by the edge-betweenness algorithm. Node colors represent relative expression levels on a gradient scale ranging from green (low) to red (high). The magnitude of the gene change is proportional to the darkness of the color. Solid line: functional interactions (FIs) extracted from pathways; dashed line: based on naïve-bayes classifier (NBC); arrow: FIs involved in activation, expression regulation or catalysis; ' $T$ ' bar: FIs involved in inhibition. (B) Colony formation of TAZ-M\#1 cells in soft agar is inhibited by Dasatinib. $(\mathrm{n}=6)(\mathrm{C})$ Quantification of mammosphere formation induced by TAZ-M\#1 cells treated in the DMSO vehicle or Dasatinib $(1 \mu \mathrm{M})$ for 6 days. Bars denote standard errors $(\mathrm{n}=6)(* * * p<0.0001)$. (D) FACS analysis of the CD44 high/CD24 $4^{\text {low }}$ population of TAZ-M\#1 cells treated with either vehicle or Dasatinib at indicated doses for 24 hours and followed by recovery for 72 hours. (E) Dose-response curve of luminal (in green) and TNBC (in red) cell lines treated with Dasatinib for $72 \mathrm{hr}$. (F) Colony formation assays of cell lines treated with Dasatinib $(300 \mathrm{nM})$ for $24 \mathrm{hr}$ and cultured for 10 days in drug-free medium. (G) Tumor formation of MDA-MB-231 cells is inhibited by Dasatinib treatment. Cells were treated with DMSO or Dasatinib (300 nM) for $24 \mathrm{hr}$ prior to mammary fat pad injection into the SCID mice. 
digital RNA sequencing and computational approaches, we present a differential network-based framework to detect biologically meaningful cancer-related genes.

Notably, we identify several developmental pathways currently under investigation as potential therapeutic targets in CSCs, such as the Hedgehog, WNT, NOTCH and Nodal/Activin pathways [34]. However, at present, it is unclear of which these pathways are direct or indirect targets of a TAZ-mediated pro-tumorigenic transcriptional program. Nevertheless, our network analyses identified a novel PDGFR- $\beta$ centered FI subcluster. The role of the PDGFR- $\beta$ in different tumor cell types and in metastasis remains poorly characterized. For example, PDGFR- $\beta$ signaling contributes to diverse tumorassociated processes, such as autocrine growth simulation of malignant cells, paracrine-stimulation of blood vessel formation and stimulation of fibroblasts and pericytes in the tumor stroma [35]. It was recently reported that mutant $\mathrm{p} 53$-induced up-regulation of PDGFR $\beta$ drives pancreatic cancer invasion and metastasis [36]. Of the intra-cellular pathways that operate downstream of PDGFR- $\beta$, the activation of the Ras and ERK MAP kinase pathway is important for cell proliferation. Actin reorganization and cell migration is dependent on PI3-kinase mediated activation of Rac, whereas PI3-kinase activation of AKT is important for cell survival. Several of these pathways are up- regulated in TAZ-expressing tumors as determined by high-density antibody microarrays (data not shown). However, direct inhibition of PDGFR- $\beta$ alone through either RNAi or PDGFR-specific inhibitors, had no effect on $\mathrm{BC}$ cell viability in vitro or TAZ-mediated tumorigenicity in vivo (data not shown).

Recent advances in computational biology have identified a network structure and phenotypic robustness that strongly suggests the higher clinical efficacy of multi target drugs as compared with individual drug targets. Adopting this basic tenet, we rationalized that combinatorial gene inhibition by the tyrosine kinase inhibitor such as Dasatinib could modulate TAZ-mediated tumorigenesis. Correspondingly, we clearly show that Dasatinib selectively targets the $\mathrm{CD} 44^{\text {high }} / \mathrm{CD} 24^{\text {low }}$ subpopulation in vitro and inhibits TNBC tumor growth in vivo. In a recent study, PDGFR- $\beta$ was shown to lie downstream of FOXC2 in BC cells induced to undergo EMT. Furthermore, the PDGFR- $\beta$ inhibitor sunitinib reduced tumor growth and metastasis of FOXC2expressing tumor cells [37], but given our findings it is most likely the effects mediated by sunitinib in these studies are a consequence of complex RTK inhibition and not direct PDGFR- $\beta$ inhibition.

Current failure in TNBC treatment usually is not due to a lack of primary response or initial induction of remission but instead relapse after chemotherapy, in which CSCs are believed to play an essential role. If the CSC hypothesis is correct, then the most appropriate cells for investigation of genes involved in TNBC may be CSCs.
Here, we demonstrate how the multi-target inhibitor, Dasatinib, selectively targets CSCs and inhibits basallike and TNBC tumor growth. Similar to other targeted cancer therapies it will be important to determine which $\mathrm{BC}$ patients are most likely to benefit from this treatment. Furthermore, future studies are required in defining optimal treatment strategies for aggressive TNBC, both in the advanced as well as adjuvant setting.

\section{MATERIALS AND METHODS}

\section{Cell culture, transfection and transduction}

MCF10A cells were cultured as described [38]. MDA-MB-231, HCC38, T47D and ZR751 cells were cultivated in RPMI-1640 medium with $10 \%$ fetal calf serum (FBS); CAL120, MDA-MB 361, MDA-MB 453 and BT474 cells were cultivated in DMEM medium with $10 \%$ FBS; CAL 51 was cultivated in DMEM medium with $20 \%$ FBS; SUM159 was cultivated in F12K medium with 5\% FBS, $5 \mu \mathrm{g} / \mathrm{ml}$ insulin and $1 \mu \mathrm{g} / \mathrm{ml}$ hydrocortisone. All media were supplemented with 100 units $/ \mathrm{ml}$ penicillin, $100 \mu \mathrm{g} / \mathrm{ml}$ streptomycin and $2 \mathrm{mM}$ glutamine. All cells were cultured in a humidified atmosphere of $95 \%$ air and $5 \% \mathrm{CO} 2$ at $37^{\circ} \mathrm{C}$. MDA-MB-231, T47D, MDAMB453, BT-474 and HCC38cells were purchased from American Type Culture Collection (ATCC, VA); Cal51, Cal120 and SUM159 were obtained from Dr. Toru Ouchi, PRCI; ZR751 and MDA-MB-361 were obtained from Dr. Andrei V. Bakin, RPCI. Transfection was performed using X-tremeGENE 9 DNA Transfection Reagent following the manufacturer's protocol (Roche). Packaging of retrovirus and lentivirus, cell transduction and drug selection were performed following standard protocols.

\section{Plasmid construction}

The human TAZ-4SA expression constructs were described previously [20].

\section{Antibodies and immunoblot analysis}

TAZ antibody was purchased from Cell Signaling Technology; YAP antibody from Santa Cruz; $\beta$-actin antibody from Upstate; fibronectin and Flag (M2) antibodies from Sigma-Aldrich; E-cadherin (CDH1), P-cadherin (CDH3), N-cadherin (CDH2), FN1 antibodies from BD Biosciences; CD24-PE and CD44-APC antibodies from Invitrogen. For protein extraction, cells were washed with phosphate-buffered saline (PBS) and collected with IP buffer: $20 \mathrm{mM}$ Tris- $\mathrm{HCl}$ (pH 8.0), $150 \mathrm{mM} \mathrm{NaCl}, 20 \%$ glycerol, $0.5 \%$ NP-40, plus $1 \square$ Complete $^{\mathrm{TM}}$ EDTA-free Protease Inhibitor Cocktail (Roche) or $1 \square$ Halt $^{\mathrm{TM}}$ EDTA-free Protease and Phosphotase Inhibitor (Thermo Scientific). Cell lysate was cleared by centrifugation at 14,000 rpm for $20 \mathrm{~min}$ 
at $4^{\circ} \mathrm{C}$. Lysate was loaded onto $4-15 \%$ MINI-PROTEAN TGX gel (Bio-Rad) with 4X SDS sample buffer. For immunoblot, proteins were transferred onto Immobilon-P membrane (Millipore), detected by various antibodies and visualized with ECL Plus Western Blotting Detection Reagents (GE Healthcare).

\section{Flow cytometry analysis}

Cells were passed through a $35-\mu \mathrm{m}$ filter, pelleted, washed in $1 \mathrm{X}$ phosphate buffered saline (PBS) $+0.5 \%$ fetal calf serum (FBS) and counted. One million cells were suspended in $1 \mathrm{X}$ PBS $+0.5 \%$ FBS and stained with anti-CD44-APC conjugate and anti-CD24-PE conjugate (BD Biosciences) for $30 \mathrm{~min}$ at $4{ }^{\circ} \mathrm{C}$. Cells were washed 3 times and then analyzed by flow cytometry.

\section{Mammosphere formation assay}

Mammosphere formation assay was performed by plating $5 \times 10^{4}$ cells in serum-free DMEM/F12 1:1 media (Gibco) supplemented with EGF (20 ng/mL) and B27 (2\%) into ultra-low attachment 6-well plates (Corning). Mammospheres were allowed to grow or in presence of drugs for 7 days. Total mammospheres greater than $100 \mu \mathrm{m}$ in diameter were counted. Each experimental group was done in triplicate and the same experiments were repeated at least three times.

\section{Cell proliferation (MTT) assay}

Two thousand to four thousand cells were plated into 96 wells plate. Plates were harvested daily. Twenty microliters of 5mg/ml MTT (3-(4, 5-Dimethylthiazol-2-yl)2, 5-diphenyltetrazolium bromide, a tetrazole) was added to each well, incubated for 3.5 hours in $37^{\circ} \mathrm{C}$, and carefully removed without disturbing the cells. One hundred and fifty microliters of MTT solvent $(4 \mathrm{mM} \mathrm{HCl}$ and $0.1 \%$ Nondet P-40 (NP40) in isopropanol) was added into each well and plate shaken at room temperature for $15 \mathrm{~min}$. Absorbance was read at $590 \mathrm{~nm}$ with a reference filter of $620 \mathrm{~nm}$.

\section{Anchorage-independent growth}

Colony formation in soft agar assay was performed as described previously [10]. Briefly, cells were suspended in a growth medium mixed with $0.4 \%$ agar and seeded into 6-well plate containing a base of $0.5 \%$ agar, at a concentration of $5 \times 10^{4}$ cells/well. Cells were incubated and colonies were read after approximately 2 weeks.

\section{In vivo tumor growth and metastasis assays}

For TAZ tumor formation, $1 \square 10^{6}$ TAZ-4SA transduced MCF10A were injected into the mammary fat pad of female NOD/SCID mice of 6-8 weeks old. For serial dilution experiments, vector control or TAZ-4SA transduced MCF10A cells, TAZ-M\#1 or TAZ-M\#5 cells in exponential growth phase were harvested and suspended in PBS (50\% matrigel), and 1 $\times 10^{6}$ (in $0.1 \mathrm{~mL}$ ) vector or $1 \times 10^{6}, 1 \times 10^{5}, 1 \times 10^{4}$, $1 \times 10^{3}$ TAZ-4SA transduced MCF10A, TAZ-M\#1 and TAZ-M\#5 were injected into the mammary fat pad of female NOD/SCID mice of 6-8 weeks old. The SCID mice were generated at the Roswell Park Cancer Institute. Tumor sizes were measured twice a week using calipers. Mammary tumor formations were also detected by the In Vivo Luminescence Imaging System. For tail vein injection, $1 \times 10^{6}$ (in $0.1 \mathrm{~mL}$ ) TAZ-M\#1 cells were injected into the tail vein of female NSG mice of 6-8 weeks old. Lung tumor formations were detected by the In Vivo Luminescence Imaging System. The care and use of animals was approved by the Institutional Animal Care and Use Committee of the Roswell Park Cancer Institute (Buffalo, NY).

\section{Cultivation of explanted tumor cells}

An explant method was used for culturing mouse tumor cells adopted from previous description [39]. Briefly, the primary mouse mammary tumors were excised, washed with growth medium (MCF10A growth medium) and then minced with scalpels to obtain pieces approximately $2 \mathrm{~mm}$ in diameter. Twelve to fifteen of these pieces were then evenly dispersed over the bottom of each 6-cm dish (Falcon Plastics) and placed at room temperature for approximately 15 minutes to allow attachment. The tissue was then covered with $5 \mathrm{ml}$ MCF10A growth medium. Cultures were incubated at $37^{\circ} \mathrm{C}$ and observed daily for evidence of growth. During the first week, the culture medium was changed only if a large amount of cellular debris was in the flasks or if the $\mathrm{pH}$ became noticeably alkaline or acidic. Care was taken to avoid dislodging the tissue explants. After the first week or after significant growth, the medium was changed at least twice a week. When cultures were confluent, cells were conservatively sub-cultured.

\section{Integrative informatics and pathway analysis}

For network and pathway enrichment analysis, we used the Reactome FI and Cytoscape plugin. Significantly enriched pathway gene sets were exported and analyzed using Enrichment Map to determine relationships between pathways [40]. To investigate functional network and gene ontology relationships, candidate genes were first categorized using the GO analysis and were performed with the DAVID software [41]. The reference population was defined by our gene study set (see above) and an adjusted $\mathrm{P}$ value of 0.05 (Benjamini and Hochberg correction was used as a threshold for the identification of significant GO terms. 


\section{ACKNOWLEDGMETNS}

We thank Drs. Ouchi Toru, Andrei Bakin for kindly sharing their cell lines and reagents with us. This work was supported by the Roswell Park Cancer Institute and National Cancer Institute (NCI) grant \#P30 CA016056 and in part by the American Cancer Society Institutional Research Grant 02-197-04; the National Cancer Institute grant \# R21CA179693 (to J.Z).

\section{Conflict of interest} interest.

The authors disclose no potential conflicts of

\section{REFERENCES}

1. Harvey KF, Zhang X, Thomas DM. The Hippo pathway and human cancer. Nature reviews Cancer. 2013; 13: 246-57.

2. Zhao B, Li L, Lei Q, Guan KL. The Hippo-YAP pathway in organ size control and tumorigenesis: an updated version. Genes \& development. 2010; 24:862-74.

3. McClatchey AI, Saotome I, Mercer K, Crowley D, Gusella JF, Bronson RT, Jacks T. Mice heterozygous for a mutation at the Nf2 tumor suppressor locus develop a range of highly metastatic tumors. Genes \& development. 1998; 12:1121-33.

4. Pan D. The hippo signaling pathway in development and cancer. Dev Cell. 2010; 19:491-505.

5. Yu FX, Guan KL. The Hippo pathway: regulators and regulations. Genes \& development. 2013; 27:355-71.

6. Lei QY, Zhang H, Zhao B, Zha ZY, Bai F, Pei XH, Zhao S, Xiong Y, Guan KL. TAZ promotes cell proliferation and epithelial-mesenchymal transition and is inhibited by the hippo pathway. Molecular and cellular biology. 2008; 28:2426-36.

7. Hao Y, Chun A, Cheung K, Rashidi B, Yang X. Tumor suppressor LATS1 is a negative regulator of oncogene YAP. The Journal of biological chemistry. 2008; 283:5496-509.

8. Zhang J, Smolen GA, Haber DA. Negative regulation of YAP by LATS1 underscores evolutionary conservation of the Drosophila Hippo pathway. Cancer research. 2008; 68:2789-94.

9. Liu CY, Zha ZY, Zhou X, Zhang H, Huang W, Zhao D, Li T, Chan SW, Lim CJ, Hong W, Zhao S, Xiong Y, Lei Q, Guan KL. The hippo tumor pathway promotes TAZ degradation by phosphorylating a phosphodegron and recruiting the SCF \{beta\}-TrCP E3 ligase. The Journal of biological chemistry. 2010; 285:37159-69.

10. Liu X, Yang N, Figel SA, Wilson KE, Morrison CD, Gelman IH, Zhang J. PTPN14 interacts with and negatively regulates the oncogenic function of YAP. Oncogene. 2013; 32:1266-73.

11. Cordenonsi M, Zanconato F, Azzolin L, Forcato M, Rosato A, Frasson C, Inui M, Montagnet M, Parenti AR, Poletti A, Daidone MG, Dupont S, Basso G, Bicciato S, Piccolo S. The Hippo transducer TAZ confers cancer stem cell-related traits on breast cancer cells. Cell. 2011; 147:759-72.

12. Silvis MR, Kreger BT, Lien WH, Klezovitch O, Rudakova GM, Camargo FD, Lantz DM, Seykora JT, Vasioukhin V. alpha-catenin is a tumor suppressor that controls cell accumulation by regulating the localization and activity of the transcriptional coactivator Yap1. Science signaling. 2011; 4:ra33.

13. Chan SW, Lim CJ, Chong YF, Pobbati AV, Huang C, Hong W. Hippo pathway-independent restriction of TAZ and YAP by angiomotin. The Journal of biological chemistry. 2011; 286:7018-26.

14. Wang W, Huang J and Chen J. Angiomotin-like proteins associate with and negatively regulate YAP1. The Journal of biological chemistry. 2011; 286:4364-70.

15. Zhao B, Li L, Lu Q, Wang LH, Liu CY, Lei Q, Guan KL. Angiomotin is a novel Hippo pathway component that inhibits YAP oncoprotein. Genes \& development. 2011; 25:51-63.

16. Hong W, Guan KL. The YAP and TAZ transcription co-activators: key downstream effectors of the mammalian Hippo pathway. Seminars in cell \& developmental biology. 2012; 23:785-93.

17. Varelas X, Sakuma R, Samavarchi-Tehrani P, Peerani R, Rao BM, Dembowy J, Yaffe MB, Zandstra PW, Wrana JL. TAZ controls Smad nucleocytoplasmic shuttling and regulates human embryonic stem-cell self-renewal. Nature cell biology. 2008; 10:837-48.

18. Johnson R, Halder G. The two faces of Hippo: targeting the Hippo pathway for regenerative medicine and cancer treatment. Nature reviews Drug discovery. 2014; 13:63-79.

19. Park HW, Guan KL. Regulation of the Hippo pathway and implications for anticancer drug development. Trends in pharmacological sciences. 2013; 34:581-9.

20. Yang N, Morrison CD, Liu P, Miecznikowski J, Bshara W, Han S, Zhu Q, Omilian AR, Li X, Zhang J. TAZ induces growth factor-independent proliferation through activation of EGFR ligand amphiregulin. Cell cycle. 2012; 11: 2922-30.

21. Al-Hajj M, Wicha MS, Benito-Hernandez A, Morrison SJ, Clarke MF. Prospective identification of tumorigenic breast cancer cells. Proceedings of the National Academy of Sciences of the United States of America. 2003; 100: 3983-8.

22. Gupta PB, Onder TT, Jiang G, Tao K, Kuperwasser C, Weinberg RA, Lander ES. Identification of selective inhibitors of cancer stem cells by high-throughput screening. Cell. 2009; 138:645-59. 
23. Petrocca F, Altschuler G, Tan SM, Mendillo ML, Yan H, Jerry DJ, Kung AL, Hide W, Ince TA, Lieberman J. A genome-wide siRNA screen identifies proteasome addiction as a vulnerability of basal-like triple-negative breast cancer cells. Cancer cell. 2013; 24:182-96.

24. Mani SA, Guo W, Liao MJ, Eaton EN, Ayyanan A, Zhou AY, Brooks M, Reinhard F, Zhang CC, Shipitsin M, Campbell LL, Polyak K, Brisken C, Yang J, Weinberg RA. The epithelial-mesenchymal transition generates cells with properties of stem cells. Cell. 2008; 133:704-15.

25. Yu M, Bardia A, Wittner BS, Stott SL, Smas ME, Ting DT, Iskoff SJ, Ciciliano JC, Well MN, Shah AM, Concannon KF, Donaldson MC, Sequist LV, Brachtel E, Sgroi D, Baselga J, Ramaswamy S, Toner M, Haber DA, Maheswaran S. Circulating breast tumor cells exhibit dynamic changes in epithelial and mesenchymal composition. Science. 2013; 339:580-4.

26. Wu G, Feng X, Stein L. A human functional protein interaction network and its application to cancer data analysis. Genome biology. 2010; 11:R53.

27. Nguyen LV, Vanner R, Dirks P, Eaves CJ. Cancer stem cells: an evolving concept. Nature reviews Cancer. 2012; 12:133-43.

28. Gnoni A, Marech I, Silvestris N, Vacca A, Lorusso V. Dasatinib: an anti-tumour agent via Src inhibition. Curr Drug Targets. 2011; 12:563-78.

29. Foulkes WD, Smith IE, Reis-Filho JS. Triple-negative breast cancer. The New England journal of medicine. 2010; 363:1938-48.

30. Zhou BB, Zhang H, Damelin M, Geles KG, Grindley JC, Dirks PB. Tumour-initiating cells: challenges and opportunities for anticancer drug discovery. Nature reviews Drug discovery. 2009; 8:806-23.

31. Frame FM, Maitland NJ. Cancer stem cells, models of study and implications of therapy resistance mechanisms. Adv Exp Med Biol. 2011; 720:105-18.

32. Kakarala M, Wicha MS. Implications of the cancer stem-cell hypothesis for breast cancer prevention and therapy. Journal of clinical oncology : official journal of the American Society of Clinical Oncology. 2008; 26: 2813-20.
33. Venkatesan K, Rual JF, Vazquez A, Stelzl U, Lemmens I, Hirozane-Kishikawa T, Hao T, Zenkner M, Xin X, Goh KL, Yildirim MA, Simonis N, Heinzmann K, et al. An empirical framework for binary interactome mapping. Nature methods. 2009; 6:83-90.

34. Visvader JE, Lindeman GJ. Cancer stem cells: current status and evolving complexities. Cell stem cell. 2012; 10:717-28.

35. Gialeli C, Nikitovic D, Kletsas D, Theocharis AD, Tzanakakis GN, Karamanos NK. PDGF/PDGFR Signaling and Targeting in Cancer Growth and Progression: Focus on Tumor Microenvironment and Cancer-Associated Fibroblasts. Current pharmaceutical design. 2013.

36. Weissmueller S, Manchado E, Saborowski M, Morris JPt, Wagenblast E, Davis CA, Moon SH, Pfister NT, Tschaharganeh DF, Kitzing T, Aust D, Markert EK, Wu J, Grimmond SM, et al. Mutant p53 Drives Pancreatic Cancer Metastasis through Cell-Autonomous PDGF Receptor beta Signaling. Cell. 2014; 157:382-94.

37. Hollier BG, Tinnirello AA, Werden SJ, Evans KW, Taube JH, Sarkar TR, Sarkar TR, Sphyris N, Shariati M, Kumar SV, Battula VL, Herschkowitz JI, Guerra R, Chang JT, et al. FOXC2 expression links epithelialmesenchymal transition and stem cell properties in breast cancer. Cancer research. 2013; 73:1981-92.

38. Debnath J, Muthuswamy SK, Brugge JS. Morphogenesis and oncogenesis of MCF-10A mammary epithelial acini grown in three-dimensional basement membrane cultures. Methods 2003; 30:256-68.

39. Giard DJ, Aaronson SA, Todaro GJ, Arnstein P, Kersey JH, Dosik H, Parks WP. In vitro cultivation of human tumors: establishment of cell lines derived from a series of solid tumors. J Natl Cancer Inst. 1973; 51:1417-23.

40. Merico D, Isserlin R, Stueker O, Emili A, Bader GD. Enrichment map: a network-based method for gene-set enrichment visualization and interpretation. PloS one. 2010; 5:e13984.

41. Dennis G, Jr., Sherman BT, Hosack DA, Yang J, Gao W, Lane HC, Lempicki RA. DAVID: Database for Annotation, Visualization, and Integrated Discovery. Genome biology. 2003; 4:P3. 